\title{
Gastric Perforation: An Unusual Complication of the Treatment of Oesophageal Atresia
}

\author{
A. W. WILKINSON,* CH.M., F.R.C.S., F.R.C.S.ED.
}

Brit. med. F., 1965, 1, 838-839

The importance of starting milk-feeding as soon as possible after major operations on newly born children is now widely appreciated. In babies with oesophageal atresia and tracheooesophageal fistula after division of the fistula and primary anastomosis, few surgeons have such confidence in the oesophageal anastomosis that they will permit even the swallowing of water within 48 hours of operation. In order to avoid continuous intravenous therapy and yet achieve a satisfactory intake of water and milk from 24 to 36 hours after operation, some form of feeding by tube is necessary, either through a fine tube passed through the nose and across the oesophageal anastomosis into the stomach at operation or by gastrostomy. For a transoesophageal tube it is usual at this hospital to use translucent polyvinyl chloride tubing (2D Portex), which is soft and limp. More than 400 children have been treated here for oesophageal atresia ; in over 300 a primary anastomosis of the oesophagus has been possible, and in most of these a Portex tube was passed across the anastomosis without complications related to the use of the tubing. This report concerns two children in whom the use of a stiffer type of tubing made of nylon resulted in perforation of the anterior gastric wall by the end of the tubing. In both cases generalized peritonitis resulted and one child died.

\section{Case 1}

The patient was a full-term male infant, birth weight $3.18 \mathrm{~kg}$. (7 lb.). The delivery was normal. He had a history of bringing up frothy saliva since birth, and when given a feed of boiled water he choked but did not go blue. On admission 36 hours after birth scattered rales were heard on both sides of chest; a catheter failed to pass to the stomach and $x$-ray examination showed it to be arrested at the level of the third thoracic vertebra; and gas was seen in the stomach and intestines. At operation 42 hours after birth a tracheo-oesophageal fistula was divided and closed, with primary anastomosis. Nylon tubing was passed across the anastomosis into the stomach. Glucose solution was administered by tube into the stomach eight hours after operation ; fluid administration continued thereafter at an increasing rate. Milk-feeds were started 24 hours after operation-5-ml. two-hourly through the tube, increased to $10 \mathrm{ml}$. and then to $15 \mathrm{ml}$. hourly by 80 hours after operation. At 88 hours after operation the abdomen was seen to be distended; bowel sounds were not heard; aspiration of the stomach produced a little milky fluid; respirations were shallow, the diaphragm was elevated, the right side of the chest moved less well than the left. Abdominal distension continued to increase very slowly, and bowel sounds were not heard during the next 12 hours. At about 105 hours distension was more pronounced and there was some greyness of the skin of the extremities. Impressions of distended intestine were seen through the abdominal wall, and a diagnosis of intestinal obstruction was made.

The abdomen was reopened through a transverse incision just above the umbilicus. On opening the peritoneal cavity large quantities of thin milky free fluid were found ; the bowel was coated with thick sticky yellowish material and bathed in milky fluid. The anterior wall of the stomach presented in the upper part of the incision, and the tube which had been passed across the anastomosis was seen projecting about $1 \frac{1}{2}$ in. $(3.8 \mathrm{~cm}$.) from the surface of the greater curvature of the stomach through a small perforation. The * Nuffield Professor of Paediatric Surgery, the Institute of Child Health
and the Hospital for Sick Children, London. end of the tube was cut off and the remainder was pulled back by the anaesthetist. The perforation in the greater curvature was closed with three interrupted silk sutures; the free fluid was removed, and the exudate was peeled off the bowel wherever this could be achieved; large quantities were removed from between the liver and the diaphragm. The wound was closed in layers without drainage ; $40 \mathrm{ml}$. of blood was transfused on the table and a further $40 \mathrm{ml}$. given during the next five hours.

The next day the patient was much better and was offered, and took, half-strength feeds of expressed breast milk. Further progress was satisfactory. Full-strength feeds were given on the third day after the second operation and further progress was uneventful. He was discharged 21 days after admission, gaining weight and taking feeds. His weight was $2.9 \mathrm{~kg}$. (6 lb. $6 \frac{3}{4} \mathrm{oz}$.). During the next month his weight rose to $3.84 \mathrm{~kg}$. ( $8 \mathrm{lb}$. $7 \frac{1}{2} \mathrm{oz}$.). During the subsequent five years his progress has been normal.

\section{Case 2}

The patient, a female infant, was delivered at 34 weeks' gestation. The pregnancy was complicated by severe hydramnios. The birth weight was $1,846 \mathrm{~g}$. (4 lb. $1 \mathrm{oz}$.). She was admitted to hospital six days after birth. Since birth an excessive amount of mucus had been brought up, but oesophageal and nasal tubes appeared to pass easily and to a normal distance. One attack of cyanosis occurred 24 hours after birth. On the second day after birth an oesophageal tube was passed; the aspirated fluid contained acid. The amount of mucus produced became less, the chest remained free of adventitious sounds, and feeding was begun on the fifth day, being accompanied by slight regurgitation through the nose at each feed. On the sixth day a radio-opaque medium, propyliodone (Dionosil), was administered through an oesophageal catheter, and $x$-ray examination revealed an oesophageal atresia, the upper pouch extending to the third thoracic vertebra, but no communication with the trachea was seen.

On admission adventitious sounds were not present. Her general condition was very good; weight $1.42 \mathrm{~kg}$. ( $3 \mathrm{lb} .2 \mathrm{oz}$.). There was slight jaundice, and much mucus in the mouth. At operation a tracheo-oesophageal fistula was divided and closed, with primary oesophageal anastomosis. A nylon tube was passed across the anastomosis into the stomach; $25 \mathrm{ml}$. of blood was transfused to replace the estimated blood loss of $20-25 \mathrm{ml}$. and was followed by $5 \%$ dextrose solution at the rate of $2 \mathrm{ml}$./hour. Subsequently halfstrength Hartmann's solution was administered at the rate of $2 \mathrm{ml}$./ hour by the tube from 15 hours after operation. Expressed breast milk was started by tube two and a half days after operation at the rate of $10 \mathrm{ml}$./hour. Progress was satisfactory until the eighth day, when the child began to vomit bile-stained fluid ; the abdomen was distended and dull on percussion, but shifting dullness was not found. $X$-ray examination of the abdomen in the upright position showed very little gas in the bowel; there were no fluid levels and no gas under the diaphragm. When opaque medium was injected down the oesophageal tube it escaped into the peritoneal cavity.

The abdomen was reopened through a transverse incision above the umbilicus and thick creamy pus was found in the abdominal cavity mixed with radio-opaque medium; thick yellow exudate was present round the stomach and on the under surface of the liver. The nylon tube was seen projecting from the greater curvature of the stomach. It was removed and the hole was closed with interrupted silk sutures; a gastrostomy was carried out and the abdomen was closed without drainage. The child made good progress at first, but then developed a bilateral bronchopneumonia and suffered frequently from cyanosis; the stomach was aspirated repeatedly, oral feeds were stopped, and intravenous infusion of 
$8 \mathrm{ml}$./hour of half-strength Hartmann's solution was maintained. There was a good deal of mucus in the mouth interfering with the airway and requiring aspiration. Ultimately a tracheostomy was made, but in spite of this the respiration did not improve and the child died on the 21st day. At necropsy there was an aspiration bronchopneumonia, the oesophageal anastomosis was satisfactorily healed, the closure of the oesophageal atresia was intact, the gastric perforation was healed, and the peritonitis was resolving. There was also a patent ductus arteriosus.

\section{Discussion}

These two anterior gastric perforations occurred at an interval of three months, but no other serious complications due to the use of indwelling oesophageal tubes have been encountered in this hospital although soft Portex tubing has been employed in over 300 children on whom a primary anastomosis has been made. Unless the tube is removed inadvertently the usual custom is to leave it in position for 7 to 10 days and then to start oral feeding. Any tubing stiffer than the limp polyvinyl chloride Portex tubing, such as that made of nylon or polyethylene, is also apt to cause ulceration at the upper and lower ends of the oesophagus as well as at the anastomosis if left in position for as long as seven days, whereas Portex tubing has not done any harm even when left for 10 days or more. The gastric perforation probably occurred on the third day after operation in Case 1 and on the sixth day in Case 2 . It is unfortunate that in Case 2 death resulted from bronchopneumonia, although after closure of the perforation the peritonitis had resolved satisfactorily.

\section{Summary}

In over 300 out of more than 400 children treated for oesophageal atresia and tracheo-oesophageal fistula it was possible to divide the fistula and make a primary anastomosis between the two pieces of oesophagus. After most of these primary anastomoses a soft Portex tube was passed across the anastomosis to the stomach to allow feeding to be started soon after operation.

In two children the anterior wall of the stomach was perforated by the use of an unusually stiff tube ; in both these children the perforation was subsequently closed, but one died later of bronchopneumonia secondary to the inhalation of vomitus after the tube had been removed.

I am indebted to Mr. D. J. Waterston for allowing me to describe the second case, which was under his care.

\section{Medical Memoranda}

\section{Conn's Syndrome and Subarachnoid Haemorrhage}

\section{Brit. med. F., 1965, 1, 839-840}

Conn's syndrome, or primary hyperaldosteronism, has been reported with increasing frequency since the first description in 1955 , shortly after the original isolation of aldosterone. The syndrome is usually due to an adenoma of the adrenal cortex, and presents in adult life with benign hypertension, with or without symptoms of hypokalaemia such as periodic weakness, paraesthesiae, or polyuria. A recent review of the literature by Conn et al. (1964) emphasizes the good results of surgical removal of the adenoma, and the importance of excluding the diagnosis in any case of hypertension.

\section{CASE Report}

A 51-year-old housewife was admitted to Chase Farm Hospital, Enfield, on 3 April 1963 with a history of severe headache for several hours, with transient loss of consciousness and subsequent drowsiness. She was known to have had a blood-pressure of 210/ 95 in 1955, but there was no other history of previous illness. On examination she was found to be semicomatose and restless, with bilateral extensor plantar responses but no other localizing signs in the nervous system and no marked neck stiffness. Her bloodpressure, which was $240 / 130$ on admission, later settled to $200 /$ 100 ; her fundi showed grade II hypertensive changes. The diagnosis of subarachnoid haemorrhage was confirmed by the presence of heavily blood-stained C.S.F. on lumbar puncture.

The electrocardiogram showed evidence of left ventricular hypertrophy, and ST depression, deep T-wave inversion, and prominent $U$ waves suggestive of hypokalaemia. This was confirmed by estimation of the plasma electrolytes, which showed a $\mathrm{K}^{+}$of
$1.8 \mathrm{mEq} / \mathrm{l}$. and mild metabolic alkalosis with a bicarbonate of $32 \mathrm{mEq} / \mathrm{l}$. (simultaneous $\mathrm{PaCO}_{2}$ from the rebreathing method was $44 \mathrm{~mm}$. $\mathrm{Hg}$ ). The blood urea and plasma sodium and chloride were all normal. The 24-hour urinary 17-hydroxycorticosteroid excretion was $21 \mathrm{mg}$. (normal 6-16 mg.) and the 17-oxosteroid excretion $4.9 \mathrm{mg}$. (normal 4-12 mg.).

Her husband denied that she had had any significant vomiting or diarrhoea, or that she was addicted to liquorice. He did say that she had suffered severe weakness of the limbs amounting almost to paralysis for several hours about 18 months previously, but there was no other story of periodic paralysis.

In the absence of any evidence of gastro-intestinal potassium loss or signs of Cushing's syndrome, it seemed likely that hyperaldosteronism was the cause of her hypokalaemia and hypertension, and after a second plasma $\mathrm{K}+$ was reported as $1.7 \mathrm{mEq} / 1$, and another E.C.G. (see Fig.) showed more marked abnormalities than the first, she was treated with intravenous dextrose and generous potassium supplements. An accurate electrolyte balance was not achieved, owing to occasional incontinence, but over four days she received $650 \mathrm{mEq}$ of $\mathrm{K}^{+}$, after which the plasma level had risen to $3.9 \mathrm{mEq} / \mathrm{l}$. and later to $4.9 \mathrm{mEq} / 1$.

With improvement in her general condition she was transferred to a neurosurgical unit on 8 April, but, owing to further haemorrhage, causing coma, angiography was not done. After her return on 18 April the coma persisted and her plasma $\mathrm{K}^{+}$was maintained within normal limits by means of intragastric potassium supplements of about $80 \mathrm{mEq} /$ day. On account of dehydration with a raised blood urea, estimation of aldosterone output was postponed, but she died suddenly on 29 April.

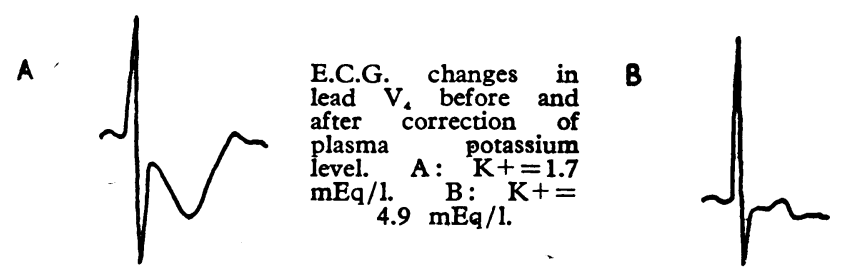

